\title{
„Im Namen der Sicherheit“ - Staatsschutzprozesse als Orte politisch-geographischer Forschung, dargestellt an Beispielen aus Gerichtsverfahren gegen Kämpfer und UnterstützerInnen der Terrororganisation „ISlamischer Staat"
}

\author{
Sarah Klosterkamp and Paul Reuber \\ Department of Geography, Münster University, Münster, Germany \\ Correspondence to: Sarah Klosterkamp (s.klosterkamp@uni-muenster.de) and \\ Paul Reuber (p.reuber@uni-muenster.de)
}

Received: 25 October 2016 - Revised: 12 June 2017 - Accepted: 22 June 2017 - Published: 5 July 2017

\begin{abstract}
Kurzfassung. In the last ten years, several uprising organizations such as the so called "Islamic State" have become a new challenge for civil societies in facing and defeating international terrorism. This paper, as part of a bigger research project, presents theoretical and methodical approaches for analyzing those organizations in the way they operate abroad and how they are connected with foreign fighters, sympathizers and supporters. By using ethnographic tools, we have observed and analyzed 14 "Islamic State"-related criminal proceedings in front of high-secured regional appeal courts. The paper presents the first results of this study in showing how German islamists are using transnational logistic networks to join or support terrorist organizations (Part 1) and gives insight into the way the logics of jurisdiction in democratic societies constitute them as "threatening subjects" (Part 2). In this way, it addresses a new approach of court-based research from the perspective of political geography.
\end{abstract}

1 Der „Islamische Staat“ und seine Verortung zwischen antizipierter Bedrohungslage und medialen wie sicherheitspolitischen Wissensdesideraten

Seit 9/11 ist islamistischer Terrorismus nicht nur zu einem zentralen Motiv internationaler Risiko- und Sicherheitsdiskurse geworden, er hat die Staaten des westlichen Militärbündnisses außenpolitisch in eine Reihe neuer Interventionskriege hineingezogen und ihnen innenpolitisch die Argumente für eine teilweise massive Verstärkung der Kontrollund Überwachungspraktiken an den nationalen Grenzen und in der homeland security an die Hand gegeben (Füller und Glasze, 2014; Nacos, 2011; Reuber, 2012; Zedner, 2012; Buzan und Hansen, 2007; Elden, 2007; Garland, 2001). Mit Anschlägen wie denen in London, Paris, Berlin und Manchester haben die TerroristInnen ihr gemeinhin als „Dschi- had "1 bezeichnetes Aufbegehren auch in die Metropolen Europas hineingetragen. Auf den Spuren ihrer Gewalttaten haben sich nicht nur die Bedrohungsängste der dortigen Bevölkerung noch einmal vervielfältigt, sondern auch die antiterroristischen Interventionen von Polizei, Militär und Geheimdiensten (Belina, 2016; Lohlker et al., 2016; Macé, 2015; Cesari, 2012).

Es sind aber längst nicht die Anschläge allein, die die öffentliche Debatte um das Sicherheitsrisiko islamistischer

\footnotetext{
${ }^{1}$ „Dschihad“ wird in den Medien, aber auch in einem großen Teil der wissenschaftlichen Literatur - philologisch falsch - meist mit „Heiliger Krieg“ übersetzt. Die richtige Übersetzung „Anstrengung" verkürzt allerdings die historische und aktuelle Situation. Die islamische Theologie unterscheidet zwischen dem „Großen Dschihad", der Anstrengung des Gläubigen, seine eigenen Schwächen zu überwinden, und dem „Kleinen Dschihad“, der auch ein bewaffneter Heidenkampf sein kann (Gemein und Redmer, 2005:223).
} 
Terrorismus beflügeln und die deutlich machen, wie sehr der islamistische Terrorismus auch in den europäischen Ländern ein Teil der kollektiven Bedrohungsdiskurse geworden ist (Ziegleder et al., 2011; Clément, 2016; Molthagen, 2016). Ebenso greifbar und sichtbar sind konkrete Alltagsphänomene, wie sie sich prominent z.B. in den Aktivitäten islamistischer Unterstützermilieus materialisieren. Im Zuge ihrer Da'wa Arbeit, der aktiven Missionierung (bspw. durch Koran-Verteilaktionen oder humanitäre Spendenveranstaltungen), rekrutieren sie nicht nur SympathisantInnenzirkel, die sich in Internetforen und Messenger-Chats organisieren, sondern auch islamistische AktivistInnen, die wahlweise als „GotteskriegerInnen“ oder „humanitäre HelferInnen“ ins Ausland gehen, um an den Kämpfen im Nahen und Mittleren Osten teilzunehmen (Abou-Taam und Sarhan, 2016:26; El-Mafaalani et al., 2016).

Während einige von ihnen in den Auseinandersetzungen des syrischen Bürgerkrieges umkommen, kehrt eine Reihe nach dem Einsatz in die europäischen Ursprungsländer zurück, inklusive dort erworbener militärischer Ausbildung, teilweise sogar mit Kampf- und Gewalterfahrung. Wie viele EuropäerInnen sich insgesamt dem bewaffneten Widerstand angeschlossen haben, ist nicht eindeutig festzustellen. Während Neumann Anfang 2015 von 500-600 deutschen StaatsbürgerInnen ausgeht, die sich dem neu ausgerufenen islamischen Kalifat angeschlossen haben sollen (Neumann, 2015:111), lagen Schätzungen der Sicherheitsbehörden im Bereich von 800-1000 (BT-Drucksache 18/8382:5). Nach jüngsten Zählungen soll es sich für Deutschland um 784 Individuen handeln, von denen seit 2012 ca. 130 gefallen und rund ein Drittel mittlerweile wieder in die Bundesrepublik zurückgekehrt sein sollen; 70 von ihnen mit aktiver Beteiligung an Kämpfen in Syrien oder im Irak oder einer entsprechenden Ausbildung (BKA et al., 2016:6ff.; Heinke, 2016).

Diese „RückkehrerInnen“ stellen nach Einschätzung der Sicherheitskräfte aus einer Reihe von Gründen ein besonderes Gefährdungsmoment der ,Inneren und Äußeren Sicherheit" der Bundesrepublik dar, entsprechend werden sie gemäß den §§89a, 89b (,Vorbereitung“ bzw. „Aufnahme von Beziehungen zur Begehung einer schweren staatsgefährdenden Gewalttat"), dem $\S 109$ h („Anwerben für fremden Wehrdienst“) oder den $\$ \S 129 a, b$ (,Bildung terroristischer Vereinigung “ im In- oder Ausland) des Strafgesetzbuches (StGB) strafrechtlich verfolgt und vor Gericht gestellt. Eine erste Welle entsprechender Prozesse gegen Mitglieder oder UnterstützerInnen des sog. „Islamischen Staates“ findet seit 2013 auch in Deutschland statt und ist seit den RAF-Prozessen die größte Serie an Terrorverhandlungen, die dieses Land erlebt hat. Während auf die Möglichkeit der Prozessbeobachtung solcher Verfahren im Normalfall vor allem JournalistInnen im Vorfeld einer entsprechenden Medienberichterstattung zurückgreifen, bietet diese, wie Studien aus den Bereichen der Socio-Legal Studies (Hoffmann, 2014; Pates et al., 2016; Hannken-Illjes et al., 2009; Scheffer, 2010), der Rechtssoziologie (Latour, 2004; Twellmann, 2016; Lautmann, 2011) und auch der Geographie zeigen (Burridge und Gill, 2016; Sylvestre et al., 2015), auch ein window of opportunity für wissenschaftliche Analysen.

Diese Möglichkeit wurde bisher für das politischgeographisch brisante Feld des internationalen Terrorismus kaum genutzt, nach Hummel et al. (2016:6) dominieren hier v.a. Analysen der Gefahrenpotentiale aus der Sicht deutscher Sicherheitsagenturen (vgl. auch Spielhaus, 2016). Für die Erkenntnisinteressen der Politischen Geographie bieten Prozessbeobachtungen dieser Staatschutzverfahren aber weit mehr, und zwar in Bezug auf zwei zentrale Dimensionen. Sie stellen einerseits die derzeit aktuellsten und detailliertesten empirischen Quellen über raumbezogene Strukturen, Ideologien und Feindbilder des islamistischen Terrorismus dar. Andererseits geben sie einen Einblick in den Umgang ,des Eigenen“, hier in Form des deutschen Rechtsstaates, mit den diesen herausfordernden „Anderen“. Beide Aspekte sind gesellschaftlich relevant, ihre forschungspraktische Umsetzung erfordert aber sowohl eine unterschiedliche theoretische Fundierung als auch - darauf aufbauend - eine nach unterschiedlichen Rationalitäten organisierte Auswertung und Interpretation des erhobenen Datenmaterials:

- Auf der ersten Ebene ermöglicht es die Analyse von Strafprozessen unter Einbindung globalisierungstheoretischer Ansätze aus der Politischen Geographie (z.B. Brenner, 2008; Massey, 1991), Teile der transnationalen politischen Geographien islamistischer Terrornetzwerke, ihrer Akteure, Netzwerke, Praktiken und Materialitäten herauszuarbeiten. Diesen Aspekt thematisiert der vorliegende Beitrag exemplarisch am Beispiel erster empirischer Ergebnisse zu den Bewegungsmustern und Praktiken von aus Deutschland stammenden Kämpfern (im derzeitigen Materialkorpus ausnahmslos männlich) inklusive ihrer lokal-global organisierten Unterstützungs- und Finanzierungsstrukturen.

- Auf der zweiten Ebene lassen sich auf Basis der Prozessbeobachtung mit Bezug auf diskurs- und identitätstheoretische Ansätze die geopolitischen Repräsentationen und Feindbilder des Eigenen und Fremden (Reuber und Strüver, 2012) rekonstruieren. Solche hegemonialen gesellschaftlichen Identitätskonstruktionen treten im Gerichtssaal sehr pointiert hervor, sie werden dort als (geopolitische) Diskurse des „Islamischen Staates“ bzw. des „islamistischen Terrorismus“ (re)produziert, schreiben sich als Deutungsfolien in Praktiken von Anklage und Angeklagten ein und tragen gemeinsam dazu bei, das Rechtssubjekt „Terrorist“ zu konstituieren.

Beide Analyseperspektiven lassen sich im Rahmen dieses eher einführend angelegten Beitrages zur Einschätzung der Leistungsfähigkeit von Gerichtsprozess-Analysen für die politisch-geographische Untersuchung des islamistischen Terrorismus nicht im Detail ausloten. Gleichwohl können durch eine exemplarisch und schlaglichtartig angelegte Be- 
Tabelle 1. Übersicht der besuchten Gerichtsverfahren im Untersuchungszeitraum Oktober 2015-April 2017.

\begin{tabular}{|c|c|c|c|c|c|}
\hline Gericht & Aktenzeichen & Angeklagte & Status & Umfang & Kürzel \\
\hline \multirow[t]{11}{*}{ OLG Düsseldorf } & III-6StS 3/15 & $\begin{array}{l}\text { Kamel Ben Yahia S. } \\
\text { Mounir R. } \\
\text { Azzedine Ait H. } \\
\text { Yusup G. }\end{array}$ & abgeschlossen & $10 / 2015-06 / 2016$ & D.01 \\
\hline & III-7StS 1/15 & $\begin{array}{l}\text { Mustafa C. } \\
\text { Sebastian B. }\end{array}$ & abgeschlossen & $10 / 2015-04 / 2016$ & D.02 \\
\hline & III-6StS 5/15 & Nils D. & abgeschlossen & $01 / 2016-03 / 2016$ & D.03 \\
\hline & III-7StS 2/15 & $\begin{array}{l}\text { Mirza Tamoor B. } \\
\text { Kais B. O. } \\
\text { Leila B. O. } \\
\text { Muhammad R. } \\
\text { Mohammed D. }\end{array}$ & abgeschlossen & 02/2016-04/2017 & D.04 \\
\hline & III-5StS 2/15 & Kerim Marc B. & abgeschlossen & 03/2016-10/2016 & D.05 \\
\hline & III-6StS 7/15 & Sabri E. D. & abgeschlossen & $04 / 2016-06 / 2016$ & D.06 \\
\hline & III-6StS 4/16 & Shahid Ilgar Oglu S. & abgeschlossen & $06 / 2016-07 / 2016$ & D.07 \\
\hline & III-6StS 2/16 & $\begin{array}{l}\text { Fadil Rudolf S. } \\
\text { Mohamed A. } \\
\text { Mustafa P. }\end{array}$ & abgeschlossen & $07 / 2016-11 / 2016$ & D.08 \\
\hline & III-5StS 1/16 & Sven L. & fortlaufend & $09 / 2016$-terminiert bis $07 / 2017$ & D.09 \\
\hline & III-6StS 5/16 & Tarik S. & abgeschlossen & $12 / 2016-04 / 2017$ & D. 10 \\
\hline & III-5StS 2/16 & Zoubir L. & abgeschlossen & 01/2017-03/2017 & D.11 \\
\hline OLG Celle & $2 \mathrm{StE} 6 / 15-3$ & $\begin{array}{l}\text { Ayoub B. } \\
\text { Ebrahim Hadj B. }\end{array}$ & abgeschlossen & $08 / 2015-12 / 2015$ & C.01 \\
\hline OLG Hamburg & 3St 2/16 & Harry S. & abgeschlossen & 06/2016-07/2016 & Н.01 \\
\hline OLG Stuttgart & $3-2 \mathrm{StE} 8 / 15$ & $\begin{array}{l}\text { Kassem E.-R. } \\
\text { Nuran B. } \\
\text { Ali F. } \\
\text { Hassan A. S. }\end{array}$ & abgeschlossen & $12 / 2015-10 / 2016$ & S.01 \\
\hline
\end{tabular}

trachtung die jeweils unterschiedlichen theoretischen Verortungen sowie die sich auf dieser Grundlage entwickelnden empirischen Möglichkeiten vorgestellt und damit verbundene Probleme andiskutiert werden (Kapitel 3.1 und 3.2). Da den meisten WissenschaftlerInnen aus dem Feld der $\mathrm{Hu}-$ mangeographie die Rationalitäten und Praktiken von Staatsschutzverfahren eher wenig geläufig sein werden, ist es zuvor aus methodologisch-methodischer Sicht hilfreich, kurz aufzuzeigen, was die Politische Geographie im Gerichtssaal an Materialien für eine Analyse des islamistischen Terrorismus vorfindet und wie diese Daten gesammelt, geordnet und ausgewertet werden können (Kapitel 2). Dabei kann gezeigt werden, dass eine entsprechende Forschung auf eine Reihe von interdisziplinär bereitgestellten Erfahrungen und Erhebungstechniken zurückgreifen kann.

\section{Der „Islamische Staat“" vor Gericht: Methodischer Zugang aus politisch-geographischer Perspektive}

Aus der Serie an Staatsschutzverfahren, die gegen islamistische SympathisantInnen und zurückgekehrte Kämp- fer aus dem Nahen und Mittleren Osten verhandelt wurden, sind 14 Verfahren beobachtet und ausgewertet worden (vgl. Tabelle 1). Hinsichtlich des Umfangs handelt es sich dabei um ca. 890 Prozessstunden, wobei die Verfahrensbeobachtungen an den Oberlandesgerichten (OLG) Celle, Düsseldorf, Hamburg und Stuttgart-Stammheim stattfanden (Erhebungszeitraum Oktober 2015-April 2017, Schwerpunkt Düsseldorf). Alle Verfahren greifen auf ein Maximum an gerichtsbezogenen Sicherheitstechnologien zurück (Hochsicherheitssäle mit Panzerglas, Einlasskontrollen, Körperscans, Maschinengewehr-Bewaffnung des Wachpersonals).

Sowohl die Auswahl des Betrachtungsfeldes als auch der Betrachtungsfokus auf das, was JuristInnen die ,Rechtswirklichkeit“" nennen, folgen einer langen Tradition gerichtsethnographischer Arbeiten, für die immer noch die von Roscoe Pound ausgegebene Devise gilt, statt law in books doch besser law in action zu studieren (Pound, 1910; Travers und Manzo, 1997; Pollner, 1987). Diese methodische Ausrichtung hat nicht zufällig Ähnlichkeiten mit den heuristischen Konzepten von Bruno Latour, der in seinen Studien über 


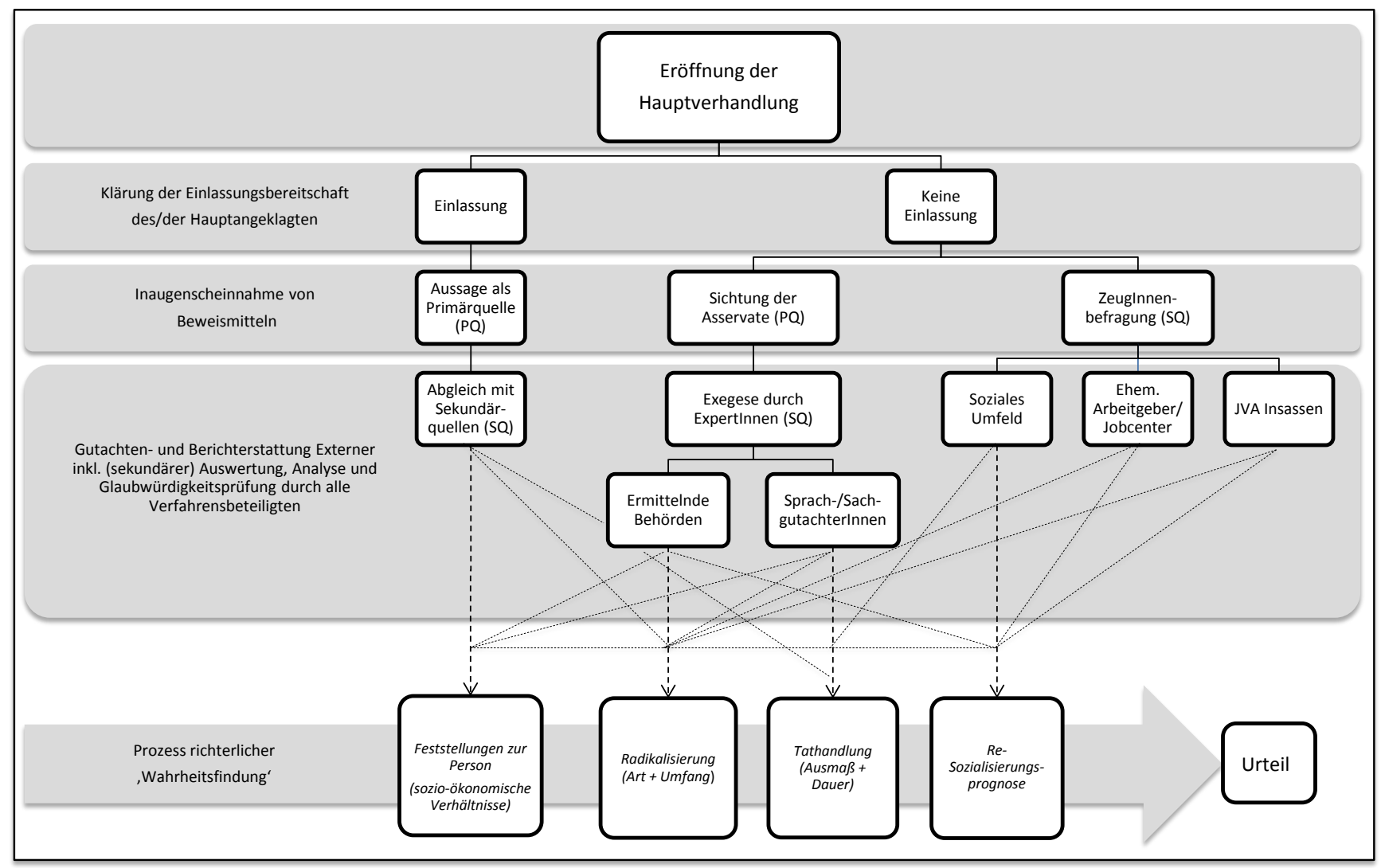

Abb. 1. Prototypischer Ablauf eines Staatsschutzverfahrens nach $\S \S 129 a+b$ StGB [Eigene Darstellung].

Science in Action (1987) ebenfalls nicht die Resultate, sondern gerade die Prozesse der Fabrikation einer Wissensordnung in den Blick nahm (Twellmann, 2016:25; Travers und Manzo, 1997:10; Atkinson, 1981).

Die Erhebungseinheit ist dabei auch für die politischgeographische Gerichtsbeobachtung der jeweilige Fall (vgl. Abb. 1). Die zeitlich und rituell stark formalisierten Verfahren mit ihren exklusiven Beteiligungsrollen stellen dabei die spezifischen (Teil-)Ordnungen des Diskurses dar, in denen er verhandelt wird (Hoffmann, 2014:287). Für die Verhandlung sind sprachliche Formen ausgebildet, in denen der Fall als „Aggregat aus kollektivem, asymmetrisch verteiltem Wissen“ (Hoffmann, 2014:290) (re-)konstruiert wird. Dies geschieht, wie Scheffer (2015) und Hoffmann (2014) in ihren Gerichtsethnographien anhand von sequentiellen Aussagenanalysen zeigen, über „Informationsverarbeitung, Perspektivierungen, Deutungen und Umdeutungen, Einblendungen und Ausblendungen, Gewichten und Präparieren, Argumente und Gegengründe" (Hoffmann, 2014:287f.). Insofern ergibt sich die Einheit eines Falles in historischer, partiell zufälliger Zusammensetzung disparater Teile, die je eine Eigenlogik haben, aber unter institutioneller Zweckbestimmung im Rahmen eines Gerichtsverfahrens zusammengeordnet erscheinen (vgl. Abb. 1), wobei sich Umfang und Wertung der einzelnen Teile je nach Gerichtsform und Rechtssystem teilwei- se erheblich voneinander unterscheiden (vgl. hierzu bspw. Hannken-Illjes et al., 2009).

Es sind gerade diese verschiedenen Teile, die in unterschiedlicher Weise Informationen für politischgeographische Forschungsfragen bereitstellen, wobei die jeweils (gerichts-)spezifischen Formen der Aussagenproduktion kritisch reflektiert werden müssen. Methodisch erschließen sie sich, da die Fall-Akten für WissenschaftlerInnen in der Regel nicht einsehbar sind, über die teilnehmende Beobachtung der Gerichtsverfahren (Lautmann, 2011:27; Vismann, 2011). Dabei wird eine Vielzahl umfangreicher und unterschiedlicher Fall-Daten inklusive ihrer Bewertung durch die Verfahrensbeteiligten zugänglich. Es sind genau diese Daten und Zeugnisse, die detailliertere politisch-geographische Forschungsarbeiten über Identitätskonstruktionen, Praktiken und Netzwerke des internationalen Terrorismus erst ermöglichen, denn bis auf die (theoretische) Möglichkeit der eigenen Feldforschung in der Konfliktregion Irak/Syrien gibt es keinen anderen empirisch zugänglichen Ort, an dem die Materialien in ähnlicher Dichte zusammenfließen. Angefangen von der Skizzierung des biographischen sowie ideologischen Werdegangs der Angeklagten sowie dem jeweiligen Familienstand, den persönlichen Vermögensverhältnissen und ihren sozialen Netzwerken, werden ZeugInnenaussagen und 
materialisierte Artefakte der Radikalisierung inklusive der daraus resultierenden Tathandlungen nach und nach in das Verfahren eingeführt. Beobacht- und erhebbar sind somit auch die „Vor- und Nebengeschichten“ der Fälle, z.B. konkrete Beteiligungs- und Unterstützungshandlungen anderer Personen oder Organisationen, die einer terroristischen Vereinigung im Sinne $\S 129$ a, b StGB oder aber $§ 89$ a StGB zugeordnet werden können.

Da die Mehrzahl der Angeklagten dieser Rückkehrer- und UnterstützerInnen-Verfahren eine Aussage - juristisch gesprochen, eine Einlassung - ablehnt, welche als Primärquelle (PQ) Eingang in die Verhandlung finden könnte, obliegt es dem Gericht, die spezifische Schuld sowie Involviertheit der/s jeweiligen Angeklagten im Rahmen einer sehr detailgetreuen Fallrekonstruktion im Rückgriff auf eine Vielzahl primärer wie sekundärer Quellen (SQ) festzustellen (vgl. Abb. 1). Ermöglicht wird dieser umfangreiche Einblick in die Beweisgegenstände (Asservate) durch das an deutschen Strafgerichten geltende Prinzip der Mündlichkeit, demzufolge nur das im Urteil berücksichtigt werden kann, was mündlich in der Hauptverhandlung vorgetragen wurde. Da die Prozesse in der Regel öffentlich sind, können die hier erhobenen Materialien prinzipiell ohne größere Einschränkungen im Forschungsprozess verwendet werden.

Abbildung 2 enthält eine Übersicht über die im Rahmen der Erhebung vorgefundenen Asservat-Arten. Unterteilt werden können sie einerseits nach Gegenständen, die nach Auffassung der Strafermittlungsbehörden und Staatsanwaltschaft (a) die konkrete Planung der Tat bezeugen, die (b) die Durchführung der Tat belegen sowie (c) Auskunft über Art und Umfang einer erfolgten Ideologisierung bzw. Radikalisierung geben. Konkret finden sich hier neben Asservaten der Durchsuchung von Haus-/Wohnungs- und Vereinsgebäuden (Reiseunterlagen, Laptops, ideologische Schriften, Videos und MP3-Formate) ebenfalls Asservate, welche aus anderweitigen polizeilichen Ermittlungen wie etwa G10Maßnahmen, Telekommunikationsüberwachungs- oder Beschattungsmaßnahmen resultieren (Abb. 2). Für eine Exegese dieser Primärquellen werden im laufenden Verfahren zusätzlich ZeugInnen- und Sachverständigenbefragungen durchgeführt (Abb. 1, z.B. Personen aus dem sozialen Umfeld der jeweiligen Angeklagten, MitarbeiterInnen involvierter Ermittlungsbehörden, IslamwissenschaftlerInnen und weitere Sachverständige, Embedded Journalists).

Vom methodischen Prozedere her wurden all diese Asservate und Befragungen in Form von dichten protokollartigen Beschreibungen des Verfahrensablaufes erfasst. Sie werden ergänzt durch manuell erstellte Skizzen eingesetzter Karten und Bildmaterialien. Da den Prozessbeobachtenden weder Video- noch Tonbandaufzeichnungen erlaubt sind, ergeben sich gerade bei multimedial präsentierten Asservaten Grenzen der Aufzeichnung im Rahmen der teilnehmenden Beobachtung. Zumindest teilweise konnten solche Aspekte durch ausführliche Postskripta (Reuber, 1993:28; Mattissek et al., 2013:197) nachgearbeitet werden, mit denen in
Prozesspausen besonders faktendichte Phasen der Verhandlung festgehalten werden. Diese sind von Bedeutung, weil gerade die multidimensionalen, ,bild-gewaltigen“ Asservate für ein tiefergehendes Verständnis der strategischen und ideologisch-religiösen Ausrichtung islamistischer terroristischer Organisationen eine wichtige Quelle bilden - inklusive der in ihnen im Zuge der Identitätsproduktion wiederkehrenden Narrationen und Ikonographien. Doch nicht nur der Gerichtssaal als solches, sondern auch Randgespräche von (und mit) Angehörigen, (selbsternannten) Gefängnisseelsorgern und AnwältInnen der Angeklagten, welche sich im Zuschauer- bzw. Eingangsbereich befinden, konnten im Zuge dieses ethnographisch informierten Feldzuganges (teilweise) erfasst werden.

Da diese Staatsschutzverfahren, wie Abb. 1 verdeutlicht, einer bestimmten diskursiven Verfahrenslogik folgen, bedarf es für eine wissenschaftliche Analyse der erhobenen Daten einer thematischen Re-Sortierung und Aufarbeitung des im Gerichtsaal als Ort autoritativer Rechtsprechung Vorgefundenen (Hoffmann, 2014:289ff.). Insofern ist der/die forschende WissenschaftlerIn neben dem Protokollieren des Beobachtbaren permanent auch mit (Re)Interpretations- und (Re)Sortierungsleistungen desselben beschäftigt.

Insgesamt, so lässt sich an dieser Stelle bereits sagen, wird durch eine teilnehmende Beobachtung aktuell laufender Gerichtsverfahren zu terroristischen Vereinigungen für die Politische Geographie eine große Bandbreite an empirischem Material für Analysen in zwei unterschiedlichen Aussagenbereichen bereitstellt: Bezogen auf konflikt- und globalisierungstheoretisch ausgerichtete, stärker praxisbezogene Fragestellungen handelt es sich um sehr konkrete Aussagen zu Netzwerken, Organisationsstrukturen und Praktiken des islamistischen Terrorismus (Kapitel 3.1). Bezogen auf diskurs- und identitätsorientierte Fragestellungen handelt es sich zum einen um Aussagen zu ideologischen Hintergründen und Formen der ,geo“-politischen Identitätsproduktion islamistischer TerroristInnen (Kapitel 3.2.1). Zum anderen lassen sich auch Aussagen zur Konstitution und Identitätsproduktion des Rechtssubjekts „Terrorist“ und entsprechender Eigen-/Fremd-Dichotomien aus der Sicht ,des Eigenen“ heraus ableiten, d.h. aus der Logik der Rechtssprechung im Rahmen von Strafverfahren vor deutschen Gerichtshöfen (Kapitel 3.2.2).

\section{Der Gerichtssaal als Ort politisch-geographischer Forschung: Beispiele aus den Prozessen gegen deutsche SympathisantInnen, UnterstützterInnen und Kämpfer des „Islamischen Staates“}

Wenn nachfolgend aus dem empirischen Material erste Ergebnisse vorgestellt werden, kann es vor dem Hintergrund der eher grundsätzlich angelegten Stoßrichtung des Beitrages nicht um eine vollständige, sondern um eine exemplarisch angelegte Betrachtung gehen. Die Beispiele lassen - 


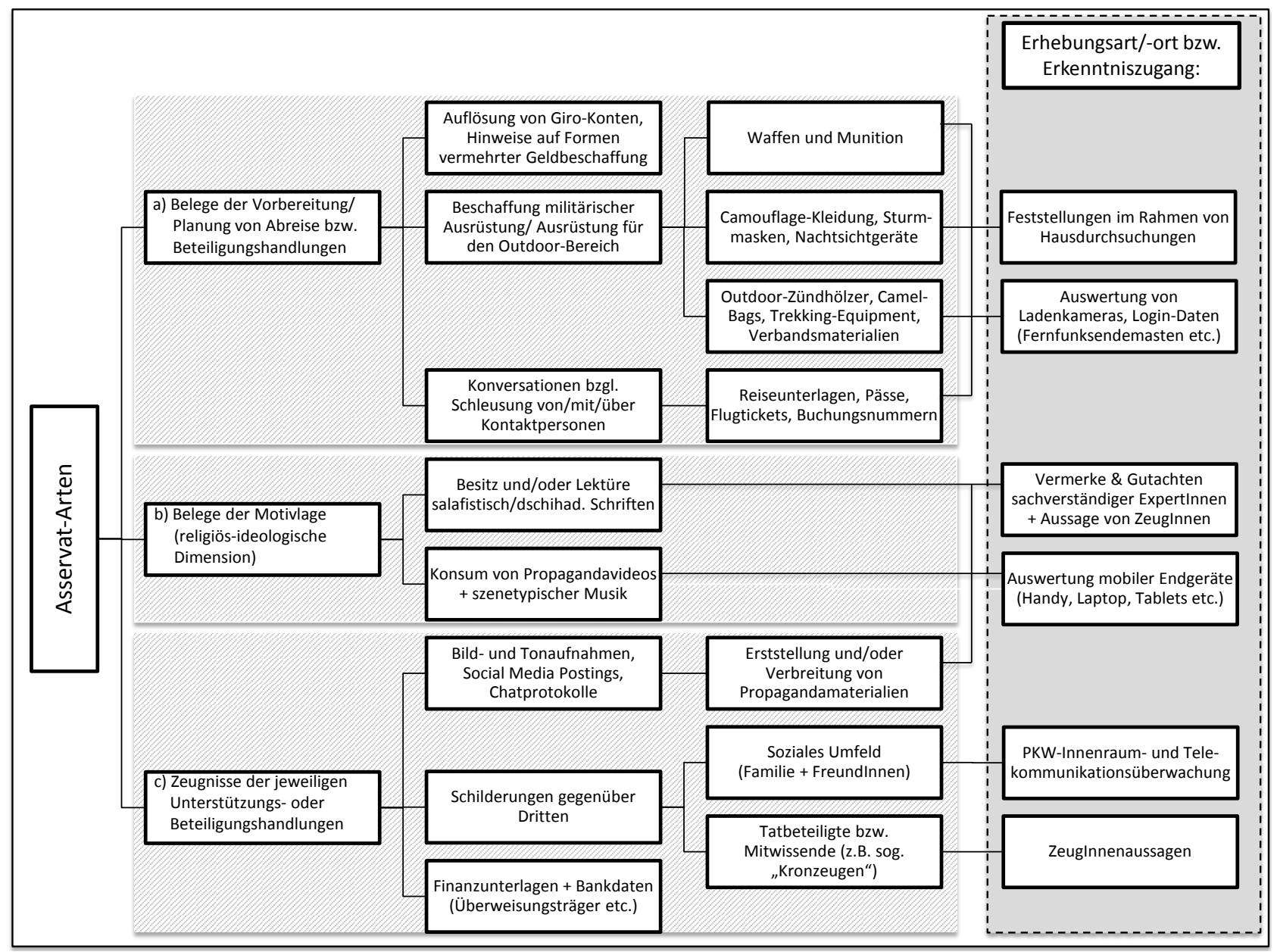

Abb. 2. Unterscheidung von Asservat-Arten in Strafprozessen [Eigene Darstellung].

mit teilweise unterschiedlicher Gewichtung - vor allem drei Aspekte hervortreten: die Anbindungen der eigenen Arbeiten an bestehende interdisziplinäre Analysen, die theoretischkonzeptionellen Verortungen, vor deren Hintergrund die erhobenen Daten aus der Gerichtsbeobachtung interpretativ erschlossen werden, sowie konkrete inhaltliche Beispiele aus den oben angesprochenen politisch-geographischen Aspekten des islamistischen Terrorismus, die auf dieser Grundlage erarbeitet werden. Dabei geht es in Kapitel 3.1 um die sozialräumliche Organisationsstruktur der terroristischen Netzwerke sowie die materiellen und finanziellen Unterstützungspraktiken, und in Kapitel 3.2 um geopolitische Identitätsproduktionen, die im Zuge der Abläufe vor Gericht bei der Konstituierung des Rechtssubjektes ,islamistische/r TerroristIn“ aktiviert werden.

\subsection{Beispiele zur Rekonstruktion von sozialräumlicher Organisationsstruktur, Netzwerken und (Unterstützungs-)Praktiken islamistischer TerroristInnen}

Will man aus den in Gerichtsverhandlungen zu Tage tretenden Informationen politisch-geographische Muster, Raumproduktionen und raumbezogene Praktiken des internationalen Terrorismus ableiten, so lassen sich als konzeptionelle Hintergrundfolien für die Interpretation der Daten auf eindrücklich passende Weise gesellschaftliche Theorien aus dem Bereich der Globalisierungsforschung heranziehen. Deren Erklärungskraft verweist darauf, dass diese Form des Terrorismus durchaus als ein Element, als eine der ,dunklen Seiten" der Globalisierung interpretiert werden kann. Bereits ein re-visiting der von Castells (2001) formulierten Netzwerkansätze erscheint hilfreich, lenken sie doch den Blick auf die Tatsache, dass es gerade die spaces of flows internationaler Waren-, Migrations- und Finanzströme sind, in denen sich wichtige Praktiken des internationalen Terrorismus erst entwickeln können. Gleichzeitig verweisen die de- 
zidiert territorial angelegten Bestrebungen des „Islamischen Staates" darauf, dass auch in dieser Variante der Globalisierung die von Castells prognostizierte Vernichtung räumlicher Organisationsformen zugunsten von Netzwerken als Erklärung zu kurz greift. Auch im Bereich des islamistischen Terrorismus ist es gerade die Kombination von Ansätzen zum Verstehen der Rolle von Netzwerkpraktiken mit Konzepten der Raumproduktion und territorialen Kontrolle, die die Vielschichtigkeit der Phänomene, Repräsentationen und Praktiken verstehbar machen. Hier führen nicht nur Eldens Bezüge (2007) zwischen Terror und Territorium weiter, es sind gerade auch die auf die Analyse globaler Ungleichheiten zielenden Varianten der Radical Geography, die z.B. im Sinne von Brenner (2008) eine spezifische Organisationsform und Machtbalance von Orten, Territorien, Grenzen und (grenzüberschreitenden) Netzwerken als machtvolle Motoren globaler Entwicklungen identifizieren. Welche spezifischen Kombinationen, Hybridisierungen und Ineinanderfaltungen von solchen „Räumen der Globalisierung“ im Sinne von Massey (1991) islamistische Terrororganisationen hier für ihre Zwecke nutzen, modifizieren oder erzeugen, soll nachfolgend an drei Beispielen aus den laufenden Prozessbeobachtungen verdeutlicht werden.

\subsubsection{Sozialräumliche Organisation: Rekonstruktion der Aufenthaltsorte und Gruppenkonstitutionen ausländischer Kämpfereinheiten}

Die in den Verhandlungen präsentierten Informationen über Aufenthaltsorte im syrischen Bürgerkrieg zeigen, dass sich die Kampfeinheiten trotz des gemeinsamen inhaltlichen Motivs ihres „heiligen“ Krieges nicht mischen, sondern dass die sozial-räumliche Herkunft als Distinktionsgrundlage für die Bildung von Einheiten dient. Ausländische Kämpfer in Syrien erhalten zunächst - und in der Regel vor dem Anschluss an die vor Gericht als „Islamischer Staat“ (IS) oder ISIS bezeichnete Organisation - eine militärische wie ideologische Unterweisung in sog. Safe Houses (D.03, 21. Januar 2016; D.08, Verweisschlüssel s. Tabelle 1), Trainingscamps (H.01, 22. Juni 2016; D.10) oder aber bei kleineren, mit dem IS koalierenden islamistisch-dschihadistischen Gruppierungen, die zumindest teilweise gemeinsame kulturelle Formatierungen aus ihren Herkunftsgebieten mitbringen (D.02, 22. April 2016; D.07). Für die Jahre 2013-2015 handelt es sich hierbei mehrheitlich, vor allem bei Ausreisenden aus Europa, um die aus vorrangig tschetschenischen, kaukasischen und libanesischen Kämpfern bestehenden Organisationen „Junud ash-Sham“ (Soldaten der Levante, kurz: JaS) sowie ,Jaish alMuhajireen wal-Ansar" (Armee der Auswanderer und Helfer, kurz: JAMWA), die auf der Seite der Aufständigen im syrischen Bürgerkrieg kämpfen. Diese gehen neben dem IS auch weitere Koalitionen ein (bspw. zur „Dschabhat Fatah asch-Scham“, Front für die Eroberung der Levante, kurz: JaN oder aber anlassbezogen mit der Freien Syrischen Armee, kurz: FSA) und bündeln sich in lokalen bis überregionalen Clustern (vgl. auch Steinberg, 2015:7).

Als Beispiel für diese territoriale Organisation der Kampfeinheiten kann anhand der laufenden Prozessverfahren die Kleinstadt Hraytan am nördlichen Rande Aleppos dienen. Sie steht durch das Fehlen staatlicher Kontrolle stellvertretend für jene Agambenschen spaces of exception, die Elden am Beispiel von al Qaida als eine der drei besonderen Beziehungen zwischen ,Terror“ und „Territorium“ bezeichnet (2007:821). Die Prozessmaterialien zeigen, dass sich die hier stationierten Einheiten vor allem aus europäischen, vornehmlich deutschen und belgischen, sowie tschetschenischen Kämpfern speisen, die von dort aus seit spätestens Juni/August 2013 auf der Seite des sog. „Islamischen Staates“ kämpfen und hier in verschiedenen Abteilungen untergebracht, organisiert und ausgebildet werden. Insgesamt wird von einer Truppenstärke von 100 Mann, verteilt auf 1015 Gruppen ausgegangen, die hier im Zentrum der Stadt stationiert sind (D.02, 3. Dezember 2015). Dass innerhalb dieser nach regionaler Herkunft sortierten Verbände in Hraytan eine weitere herkunftsbezogene Binnendifferenzierung erfolgt, zeigt das sog. „Deutsche Haus“, das gegenüber eines unterirdischen IS-Krankenhauses gelegen ist. Hier hielten sich für den Zeitraum von August 2013 bis September 2014 fünf Personen aus Nordrhein-Westfalen sowie ein Kämpfer aus Baden-Württemberg auf (D.02, 20. November 2015; D.09; D.11). Sie nahmen dort neben Tätigkeiten im Bereich Nachschub/Logistik (z.B. Küchendienste und Transport von Verpflegung an die Frontlinie) nach Auffassung des zuständigen Strafsenats am OLG Düsseldorf teilweise auch selbst an Kampfhandlungen teil (D.02, 22. April 2016).

Auch wenn solche territorial basierten Muster den Umgang mit ausländischen Kämpfern prägen, werden die lokalglobalen Räume dieses Krieges doch stark durchdrungen und ermöglicht durch netzwerkartige Strukturen. Dies zeigt sich bezogen auf diese Gruppe beispielsweise an der tragenden Rolle internetbasierter Nachrichtenkanäle und sozialer Medien wie WhatsApp, Telegram oder Facebook. Sie sind nicht nur bei der transnationalen Verbreitung entsprechender Propagandanarrationen bedeutend, sondern maßgeblich auch bei der internationalen Vernetzung (Abou-Taam und Sarhan, 2016; Lohlker et al., 2016), bei der Rekrutierung von SympathisantInnen und Kämpfern (Ben Slama, 2016) und bei deren Kontakten mit ,der Heimat“, über die die Gerichtsmaterialien detailliert Auskunft geben. Ihre Funktion reicht bis zu transnational vernetzten Benachrichtigungssystemen im Falle des Todes ausländischer Kämpfer. Zwar wird die Nachricht selbst nicht elektronisch, sondern persönlich überbracht, wobei Boten bestückt mit einem persönlichen Gegenstand des Kämpfers die Mütter der Gefallenen aufsuchen (D.02, 4. Dezember 2015). Die Boten selbst jedoch, ihre Botschaft und auch die Übermittlung der Zieladressen in der Bundesrepublik werden im Hintergrund via Mobiltelefon zentral aus Syrien oder der Türkei gesteuert (ebd., zur Bedeutung des Mobiltelefons: Buschauer, 2010:294f.). Ent- 
sprechende Praktiken lassen sich im Zuge der Verhandlungen vor Gericht aus Telefonüberwachungsprotokollen, ausgewerteten Chatverläufen, Sendemastinformationen und ZeugInnenaussagen der Mütter getöteter Kämpfer rekonstruieren und protokollieren.

Sie sind in dieser Form eines von vielen Beispielen, die die wechselseitige hybride Verschmelzung territorialer und netzwerkartiger Formen in diesen transnationalen Gewalträumen dokumentieren und hier v.a. auch die spezifische Rolle „,von Netzwerken und Vernetzung als Grundlage eines alternativen, topologischen Modus sozial-räumlicher Organisation“ (Brenner, 2008:76) verdeutlichen.

\subsubsection{Art und Umfang der Ausrüstung sowie Strategien und Routen der Materialbeschaffung}

Ein weiteres Beispiel für die Verfaltungen territorialer und netzwerkartiger Strukturen im Feld des islamistischen Terrorismus zeigt sich im Bereich der Logistik und des Nachschubs, z.B. wenn es um die Versorgung ausländischer Kämpfer mit spezifischem Material für den Aufenthalt und den Kampf in Syrien geht. Auch diese Muster lassen sich einschließlich ihrer räumlichen und personellen Beschaffungswege aus den im Rahmen der Gerichtsverhandlung vorgelegten Materialien empirisch rekonstruieren. So zeigt sich beispielsweise - erneut im Falle der oben bereits beschriebenen Kämpfereinheit - wie ein gesondertes Mitglied der Gruppe, Ismail I., gemeinsam mit seinem Bruder und einer weiteren Person beauftragt wurde, unter dem Vorwand einer gemeinsamen Türkei-Trekkingtour in Deutschland erworbene Nachtsichtgeräte, Thermo-Kleidung sowie Medikamente und Verbandsmaterialen zu den Kämpfern im „Deutschen Haus" nach Aleppo zu bringen. Anhand entsprechender Beweisstücke (z.B. Notizbuch mit „Einkaufsliste“ sowie zugeordneter Kämpfernamen) und Zeugenaussagen (D.09, 14. Dezember 2016; D.02) ließen sich Nachschubrouten, Funktionsweisen und Strategien der Materialbeschaffung dieser Kampfeinheit rekonstruieren. Weitere vor Gericht in Augenschein genommene Listen geben Auskunft über benötigte Medikamente und medizinische Geräte zur Behandlung von Kampfverletzungen, die in Deutschland beschafft und nach Syrien geschleust werden sollten, und liefern schließlich neben den Beschaffungsgegenständen auch Auskunft über Namen und Kontaktdaten schleusender Mittelsmänner in der Türkei, die gleichzeitig auch in anderen Gerichtsverfahren auftauchten (D.11; D.01).

Bündelt man diese fallbezogenen Erkenntnisse mit Beobachtungen in weiteren Gerichtsverfahren, so treten nicht nur die komplexeren Raum-Netzwerkstrukturen dieser Logistiken zu Tage, sondern auch Umfang und Ausmaß der Transfers. In einem Verfahren am OLG Stuttgart werden die Angeklagten beschuldigt, 7500 Stiefel, 6000 Militärparka und 100 Militärhemden im Wert von über 130000 Euro an die zweitgrößte Rebellenmiliz des syrischen Bürgerkrieges, der „Harakat Ahrar al-Sham al-Islamiya“ (Islamische Bewegung der Freien Männer Großsyriens, kurz: Ahrar al Sham) geliefert zu haben (S.01, 22. September 2016). Auch die Überführung von Kraftfahrzeugen - insbesondere in Deutschland ausrangierte Krankenwagen- und Feuerwehrfahrzeuge - in das syrische Kriegsgebiet im Zeitraum von 2013-2014 wurden in laufenden Verfahren am OLG Düsseldorf daraufhin geprüft, inwiefern sie als Unterstützungsleistungen terroristischer Vereinigungen zu werten sind (D.04, 06. April 2017; D.09).

Gemeinsam sind solche Befunde über ihre empirische Bedeutung hinaus auch konzeptionell in der Lage, ein weiteres Mal den Blick für die vernetzten Geographien des im Rahmen der Globalisierung immer wichtiger werdenden und immer komplexer organisierten „Logistiksektors“ zu schärfen. Sie vertiefen unsere Vorstellung davon, wie im Bereich von Nachschub und Logistik des international organisierten islamistischen Terrorismus vielfältige und komplexe „,soziale Kräfte transversal über Orte, Territorien und Scales hinweg verbunden werden“ (Brenner, 2008:77). Dabei können Kerngedanken von Cowens Deadly Logistics (2014) hilfreich sein, nicht zuletzt ihr Plädoyer für ein ,,actively rethinking security “ in diesem Feld (ebd.: 81). Gleichzeitig weisen die Gerichtsbefunde in Bezug auf die politische Brisanz der räumlichen Organisation von Logistik im Kontext internationaler Gewaltkonflikte, Gewalträume und Netzwerkstrukturen über diese hinaus (vgl. auch Whatmore und Thome, 1997:285; Leitner, 2004:248f.).

\subsubsection{Formen und Struktur der Entlohnung, Finanzierung und Geldbeschaffung ausländischer terroristischer Organisationen durch deutsche StaatsbürgerInnen}

Ein weiterer, für das Verständnis der Praktiken islamistischer Terrornetzwerke zentraler Aspekt betrifft deren Finanzierung. Auch hier zeigen die im Rahmen der Gerichtsverfahren präsentierten Asservate, wie in den hybriden Geographien der Finanzflüsse des islamistischen Terrors Praktiken zusammenwirken, die einerseits die territorialen Logiken spezifischer sozialer Finanz- und Unterstützungsmodi in bestimmten Ländern nutzen, und andererseits die netzbasierten und digitalisierten Finanzflüsse für ihre Zwecke instrumentalisieren. Konkret lassen sich aus den präsentierten Asservaten nicht nur die Art und der Umfang der Beteiligung deutscher StaatsbürgerInnen herausarbeiten, sondern auch die spezifischen Strategien der Geldbeschaffung, die analogen und digitalen „Wege des Geldes“ bei Finanztransfers durch ausländische UnterstützerInnennetzwerke (bspw. in Deutschland und der Türkei inklusive der beim Transfer des Geldes beteiligten Mittelsmänner und ihrer Verschleierungstaktiken) sowie Formen und Strukturen der Entlohnung deutscher Kämpfer in Syrien. Diese Aspekte sollen nachfolgend an drei Beispielen aus dem Feld der Finanzierung und Geldbeschaffung ausländischer terroristischer Organisationen durch deutsche StaatsbürgerInnen verdeutlicht werden: 
am Missbrauch staatlicher Unterstützungsleistungen (D.01, 17. Dezember 2015; D.02), an der Sammlung von Spenden (D.04, 6. April 2017; D.01) sowie an kriminellen Methoden wie Diebstahl, Einbrüchen oder Raubüberfällen (D.01, 19. November 2015; D.03).

Bezogen auf die Rekonstruktion von Praktiken der missbräuchlichen Verwendung staatlicher Unterstützungsleistungen kann im umfassenden Rückgriff auf Bankdaten nachgewiesen werden, wie die Finanzierung von Aktivitäten der Angeklagten mithilfe zweckentfremdeter staatlicher Unterstützungsleistungen funktioniert. Zum Transfer des Geldes verfügen einige der Angeklagten über eine Vielzahl fremder EC-Karten (inklusive der zugehörigen PIN-Nummern), die auf unterschiedlichen Wegen angeeignet oder untereinander ausgetauscht werden (D.01, 3. Dezember 2015; D.02). Die Ausnutzung territorial basierter Praktiken staatlicher Unterstützung greift hier mit der Anonymität digitaler Praktiken der Finanzwirtschaft ineinander, wenn die Angeklagten auf diese Weise auf Bankkonten von Familien (teilw. auch von Einzelpersonen) zugreifen konnten, die sich zum gegebenen Zeitpunkt nachweislich nicht mehr in Deutschland aufhielten, die aber nach wie vor EmpfängerInnen deutscher Sozialleistungen waren und diese somit zur Finanzierung der terroristischen Aktivitäten zweckentfremdet wurden. In ähnlicher Weise wurden Kindergeldzahlungen sowie Zahlungen von Arbeitslosenbeihilfe von den Konten mehrerer Familien aus dem Raum Aachen in Nordrhein-Westfalen in einem fünfstelligen Bereich zwischen August 2013 und Juni 2014 nach Syrien oder in die Türkei transferiert. Sofern für diese staatlichen Unterstützungsleistungen Neuanträge erforderlich waren, wurden kurzzeitige Aufenthalte der sich eigentlich im Ausland aufhaltenden Kontoinhaber bzw. LeistungsempfängerInnen in Deutschland einkalkuliert (D.01, 8. Juni 2016).

Ein anderes Beispiel für die Finanzierung sind Spendensammlungen. So zeigt die Beobachtung der Verhandlungen, wie mit Hilfe verschiedener von Seiten der Ermittlungsbehörden erhobener Beweisstücke angenommen werden kann, dass z.B. im Rahmen von als karitativ deklarierten Galaveranstaltungen von dort anwesenden einschlägigen Vereinen wie „Helfen in Not“, „Ansaar International“ oder von den in Nordrhein-Westfalen operierenden Da'Wa Gruppierungen Geldspenden gesammelt und anschließend digital via WesternUnion oder auf analoge Weise via Boten über die Türkei nach Syrien transferiert werden und so bspw. zur Rückführung oder Rehabilitierung verwundeter oder in der Türkei festgesetzter „Brüder“ in das Krisengebiet beitragen (D.01, 3. Dezember 2015).

Ein dritter Zweig der Finanzierung speist sich aus kriminellen Aktivitäten, zu denen Betrugsdelikte (z.B. der Verkauf des Inhalts von bei Zustelldiensten wie DHL und Hermes entwendeten Paketen (D.01, 3. Dezember 2015) oder das Abschließen finanziell nicht gedeckter Mobilfunkverträge (D.03, 20. Januar 2016)), aber auch Diebstähle und Einbruchsdelikte gehören (D.04, 10. Februar 2016).

\subsection{Identitätskonstruktionen des „Eigenen“ und des „Fremden“ im Rahmen deutscher Staatsschutzverfahren}

Die zweite aus Sicht der Politischen Geographie zentrale Erkenntnisebene bei der empirischen Beobachtung von Staatsschutzverfahren im Umfeld islamistischer Terrornetzwerke betrifft das Feld der geopolitischen Identitäten. Solche politisch-geographischen Raumproduktionen sind im Feld des internationalen Terrorismus nicht nur deswegen so zentral, weil die Terroristen selbst das Eigene und das Fremde in Form einer kultur-räumlichen Kopplung herstellen (bis hin zum ,islamischen Staat“), sondern weil solche Repräsentationsweisen im Zuge der Verhandlungen sowohl von den Angeklagten als auch seitens des deutschen Staates und seiner Gerichtsbarkeit vielfältig aktiv produziert und reproduziert werden. Sie werden damit zu einem zentralen Element für Prozess und Urteilsfindung. Auf diese Weise adressieren sie auch die Frage, inwieweit und mit Bezug auf welche ,kulturräumlich" konnotierten Weltbilder und Werteordnungen die Angeklagten als ,islamistische TerroristInnen“ verortet werden. Während also aus strafrechtlicher Sicht vor allem die Fragen nach einzelnen Bestätigungs- und Unterstützungshandlungen für das Verfahren selbst relevant sind, sind für eine politisch-geographische Analyse die hier sehr dual und apodiktisch auftretenden geopolitisch-ideologischen Identitätszuschreibungen von Interesse, die sich auf der Grundlage diskurs- und identitätstheoretischer Ansätze beobachten und interpretieren lassen.

Dieser gerade mit Blick auf gesellschaftliche Konfliktund Risikodiskurse wichtige Aspekt der juristischen Verfahren ist bisher kaum wissenschaftlich bearbeitet worden. Die derzeit vorliegenden Beiträge nehmen zum einen straf- sowie staatsrechtliche Dimensionen deutscher Gerichtsverfahren im Zuge der durch den war on terror hervorgebrachten Versicherheitlichungstendenzen westlicher Gesellschaften in den Fokus (Korf, 2009; Zedner, 2012; Buzan und Hansen, 2007; Cesari, 2012), sie analysieren zum zweiten allgemeiner rechtliche und normative Aspekte der Techniken des Polizierens (Belina, 2016; Klamt, 2007) und beschreiben allgemeiner die im Rahmen der Externalisierung dieser Verfahrensweisen herausbildenden spaces of exception am Beispiel von Guantánamo oder der Festsetzung und Internierung von Gefangenen (Gregory, 2006, 2017; Hannah, 2006). Erst in Ansätzen sind die in den Strafverfahren zu Tage tretenden spatial tactics (Sylvestre et al., 2015) und geopolitischen Imaginationen selbst (inklusive ihrer Reifikation von Freund/Feindbildern) sowie die sich auf dieser Grundlage entwickelnde Konstituierung von Rechtssubjekten und (prekären) Positionalitäten (Burridge und Gill, 2016) zum Gegenstand wissenschaftlicher Betrachtung geworden. 


\subsubsection{Ausgangspunkte und empirische Daten für die (Re-)Konstruktion geopolitischer Identitätsproduktionen vor Gericht}

Will man sich diesem Thema im Zuge der Prozessbeobachtung empirisch nähern, dann eignen sich als Ausgangspunkte für die Auswertung der dort gewonnen Daten diskurs- und identitätstheoretische Ansätze, die sich in der Tradition von Foucault (1981) und Laclau und Mouffe (1985) mit den politischen Machtaspekten gesellschaftlicher Identitätsdiskurse, Leitbilder und Risikokonstruktionen auseinandersetzen. Bezogen auf die identitätsstiftende Verbindung von Religion und Raum, wie sie im Umfeld des islamistischen Terrorismus spätestens seit dem 11. September 2001 sehr prominent geworden ist, können zur Konkretisierung Arbeiten aus dem breiten Feld der Orientalismus-Debatte (Said, 1978; Gregory, 2013) und der Kritik der Huntington'schen KulturkampfThesen herangezogen werden (Reuber und Wolkersdorfer, 2002; Husseini de Araújo, 2011; Reuber, 2012:184ff.).

Empirische Befunde zu diesem inhaltlichen Komplex treten in den Staatsschutzprozessen in vielfältigen Formen zu Tage. Sie finden sich in den im Rahmen des Verfahrens vorgelegten Fall-Daten (z.B. Propagandavideos, Fotos, dschihadistische Audiodateien, Manifeste und Schriften). Neben den Befunden aus vorgelegten Asservaten sind auch der Habitus und die Praktiken der Angeklagten Gegenstand bzw. Bezugspunkte entsprechender Identitäts-Verortungen: Vor Gericht geht es z.B. um die Länge und Dichte der Bärte; um ihre religiösen Praktiken (Art, Häufigkeit und Ausgestaltung ihrer täglichen Gebete, abgefragt von den Mitinsassen der jeweiligen Justizvollzugsanstalten, D.01, 10. Dezember 2015; D.10, 8. Februar 2016) oder um ihre Verweigerungshaltungen vor Gericht (z.B. Missachtung oder Umgehung prozessualer Rituale, D.10, 22. Dezember 2016; D.02). Auch das demonstrative Hochhalten von Koranexemplaren oder politischen Symbolen (D.02, 22. April 2016) kann ebenso wie das Insistieren auf simultane Dolmetscherdienste (trotz Kenntnissen der deutschen Sprache und zugleich ohne Nutzung der notwendigen Technik, D.01, 17. März 2016; D.04) als identitätspolitische Verortung interpretiert werden. Weitere Belege bezieht das Gericht z.B. aus vorgelegten Gruppenfotos der Angeklagten gemeinsam mit islamistisch-dschihadistisch orientierten Brüdern, auf denen sie mit entsprechenden Flaggen, Gesten und Symboliken posieren (D.08, 29. September 2016; D.02), aus (selbstproduzierten) islamischen Ermächtigungsgesängen, anāš̌̀d genannt, die sich auf den MP3-Playern und Mobiltelefonen der Angeklagten befinden (D.06, 4. Mai 2016; D.01) sowie aus der Produktion (H.01, 23. Juni 2016; D.06), der Verbreitung oder dem Besitz von Propagandavideos ausländischer terroristischer Vereinigungen.

Bereits diese wenigen Beispiele machen aber auch deutlich, dass für die Konstruktion geopolitischer Identitäten vor Gericht neben den Angeklagten die diskursiven Rahmungen und Praktiken der auf deutscher Seite in den Prozess involvierten Verfahrensbeteiligten von großer Bedeu- tung sind (Staatsanwaltschaft, Ermittlungsbehörden, Sprachund Sachverständige, rechtssprechende Strafsenate). Deren identitäts(re-)produzierender Blick wird vor allem in solchen Verfahren sichtbar, wo die Angeklagten die Aussage verweigern. Denn in diesem Fall müssen sie im Sinne der Wahrheitssuche aus der „grauen“ Vielfalt der Indizien eine ,schwarz-weiße“ Optik der Beurteilung produzieren, was sich bis in die richterlichen Urteilsbegründungen hinein verfolgen lässt. Gerade diese nehmen häufig explizit auf die vorgebrachten geopolitischen Identitätskonstruktionen Bezug und liefern damit selbst ein Datenmaterial, in dem sich kollektive Subjektivierungsformen des Eigenen und des Fremden spiegeln. Hier wird noch einmal brennglasartig deutlich, dass sich das Gericht zwar in der „Aufdeckung des Falschen auf die [beteiligten] Parteien“ (Hoffmann, 2014:293) verlässt, aber auch selbst im Sinne einer Metasubjektivierung ,unausgesprochenes Weltwissen, Wissen um Normalitäten (normale Abläufe, typische Eigenschaften, Dispositionen etc.)“ (ebd.) hervorbringt. In diesem Sinne fungiert das Gericht im Auftrag der hegemonialen rechtsstaatlichen Ordnung als eine Institution der identitären Grenz-Ziehung und dies umso mehr in territorial gerahmten, d.h. geopolitisch unterlegten Prozessen, in denen die ,Innere

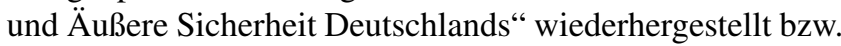
gewährleistet werden soll.

\subsubsection{Achsen der Identitätsproduktion im Gerichtssaal - eine erste Systematisierung}

Systematisiert man die empirischen Beobachtungen, so zeigt sich, dass die Staatsschutzverfahren gegen Mitglieder oder UnterstützerInnen des sog. „Islamischen Staates“ im Sinne der Logik der Urteilsfindung vor allem in zwei Dimensionen zu einer Art von Identitäten-Produktions-Maschine werden. Es geht zum einen um den Grad der Radikalität der religiösen Überzeugungen der Angeklagten (islamisch vs. islamistisch), zum anderen um die Frage, ob der Angeklagte als ein die staatliche Ordnung bedrohender ,Terrorist“ angesehen werden muss (kein Terrorist vs. Terrorist). Im Verlauf des Prozesses und abhängig von unterschiedlichen SprecherInnenpositionen können die beiden Dimensionen was die ,Verortung“ der Angeklagten angeht - in ein spannungsvolles Wechselverhältnis mit unterschiedlichen Kombinationsmöglichkeiten treten.

Im Rahmen der Abwägungen zur Feststellung der angenommenen Radikalität religiöser Überzeugungen treten alte Abgrenzungsdiskurse aus den „Risiko-Archiven“ der westlichen Moderne hervor (Foucault, 1981; Reuber und Strüver, 2012). Sie werden - ungeachtet aller wissenschaftlich-poststrukturalistischen Dekonstruktionsarbeit der Postmoderne-Debatten - im Gerichtssaal herangezogen, wenn sie nicht nur das Eigene vom Fremden, sondern die signifikant „Bedrohlichen“ gegenüber den „MitläuferInnen“- oder auch den „IrrläuferInnen“ - abgrenzen (müssen), wenn das Gericht zu prüfen sucht, wie islam-istisch sich die 


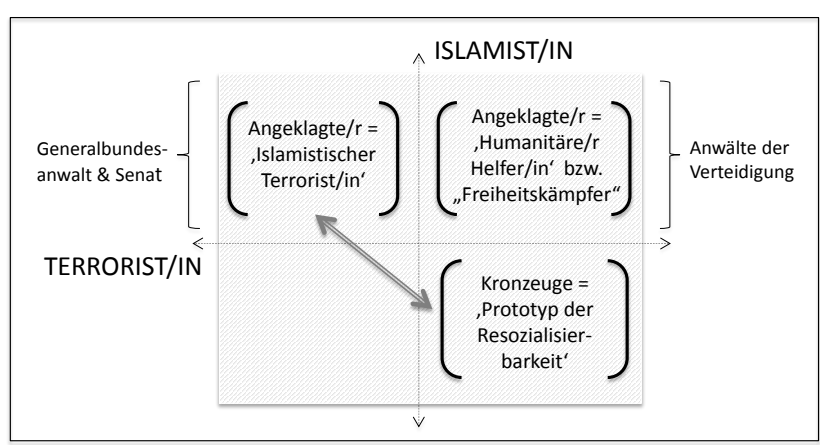

Abb. 3. Koordinatensystem einer doppelten, zwischen Fremdund Eigenzuschreibung zu verortenden, „geo“-politischen Identitätskonstruktion im Zuge deutscher Staatsschutzverfahren nach $\S \S 129 \mathrm{a}+\mathrm{b}, \S 89$ StGB [Eigene Darstellung].

Identitätskonzepte und Praktiken der Angeklagten darstellen. Die dabei gestellten Fragen und Abwägungen machen an vielen Stellen deutlich, wie sehr die Be-Urteilung hier auch auf optische Stereotypisierungen und Indizien zurückgreifen muss, deren Nähe zu klassisch orientalistischen Dichotomien manchmal frappierend wirkt: Können von außen beobachtbare Merkmale wie z.B. ein Bart gewisser Länge, bestimmte Formen der Kleidung, oder bestimmte religiöse Praktiken bereits als Indizien für den Beginn oder gar die Vollendung einer islamistischen Radikalisierung interpretiert werden? (D.01, 26. November 2015; D.10).

Die zweite oben angesprochene Achse der Identitätszuschreibung markiert die Unterscheidung, ob ein/e Angeklagte/r als „TerroristIn“ einzustufen ist oder nicht: „Ab wann gilt eine Tat oder Gruppierung als terroristisch?" (D.02, 20. November 2016). Diese Differenzierung ist aus politischgeographischer Perspektive relevant, weil das Phänomen des Terrorismus auch gegen ,den Staat“, d.h. hier gegen die auf dem Staatsgebiet Deutschlands etablierte Gesellschaft und ihre Ordnung gerichtet ist (vgl. Nacos, 2011:17ff.; Korf, 2009). Auch diese Zuschreibung, wer in die juristische Kategorie „TerroristIn“ fällt, erfolgt am Ende in der gerichtlichen Logik binär im Sinne eines „entweder/oder“".

Die systematische Auswertung der empirischen Gerichtsbeobachtungen kann zeigen, in welcher Weise diese beiden Achsen der Identitätszuschreibung im Prozessgeschehen miteinander kombiniert werden und in Wechselwirkung treten. Sie bilden das Koordinatensystem einer doppelten ,geo“-politischen Identitätskonstruktion, innerhalb derer die Angeklagten im Verlauf des Prozesses aus unterschiedlichen SprecherInnenpositionen heraus unterschiedlich verortet werden, was sich auch graphisch visualisieren lässt (Abb. 3). Die $y$-Achse symbolisiert den Grad der religiös-ideologischen Aufladung der verhandelten Subjekte, die $x$-Achse repräsentiert die Verortung hinsichtlich der „Rechtstreue“ gegenüber der territorial verfassten WirGemeinschaft des deutschen Staates. Mit dieser Betrach- tungsweise lassen sich nach ihren identitären Verortungen drei Typen von SprecherInnenpositionen unterscheiden:

- Für die SprecherInnenposition der Generalbundesanwaltschaft stellt die Kategorie „IslamistIn“ auf dem äußeren Pol der $y$-Achse den Ausgangspunkt der Verortung dar, denn sie ist juristische Grundlage der Verhaftung, die es dann - gemäß der in Deutschland geltenden Offizialmaxime - im Prozess nachzuweisen gilt (Hoffmann, 2014:289). Auf der $x$-Achse der Rechtstreue im Sinne der rechtspolitischen Ordnung des Nationalstaates markiert die Verortung als „TerroristIn“ eine Positionierung, in der sich das angeklagte Subjekt von der hegemonialen, rechtstaatlich verfassten demokratischen Ordnung weit entfernt hat und diese aus Sicht der anklagenden Instanz ablehnt, wenn nicht gar bedroht (D.08, 9. Juni 2016).

- Die SprecherInnenpositionen der Angeklagten und ihrer RechtsvertreterInnen nehmen eine andere Positionierung in diesem identitären Koordinatensystem ein. Dazu müssen sie sich - sofern sie nicht die Aussage verweigern - an den gleichen Identitätskonstruktionen und -markern ,abarbeiten“. So argumentiert die Verteidigung mehrheitlich in die Richtung, dass eine ideologische (d.h. islamistische) Aufladung zwar nicht in jedem Fall von der Hand zu weisen sei ( $y$-Achse), dass jedoch gleichzeitig die Radikalität der Haltung ( $x$-Achse) in Frage zu stellen ist (D.10, 29. März 2017; H.01). Quantität und Qualität täglicher Gebete, Musik- und Videovorlieben sowie die Länge der Bärte oder des Niqab ihrer MandantInnen seien keine hinreichenden Indizien für die von Seiten des Senats sowie der Generalbundesanwaltschaft zugeschriebene Radikalität, sondern würden vielmehr vorführen, wie Andersartigkeit mit vermeintlicher Gefährlichkeit gleichgesetzt werde (D.04, 30. März 2017; D.06). Aus dieser identitären Verortung leitet die Verteidigung schließlich eine Argumentation ab, die die Taten der Angeklagten nicht als Beihilfe oder Mitgliedschaft in eine terroristischen Vereinigungen interpretiert sehen möchte, sondern entweder als „Freiheitskampf“ und/oder als eine Art ,humanitärer Hilfe“ für die „Glaubensbrüder" in den Krisenregionen des Nahen Ostens (im Sinne einer ,,muslimischen Solidargemeinschaft"), welche als Reaktion auf die als nur unzureichend erscheinenden Interventions- und Hilfsbemühungen der westlichen Staatengemeinschaft gelesen werden müsse (D.02, 17. März 2016; D.08). Allenfalls handle es sich um den Irrweg ,gescheiterter“, zumeist noch ,jugendlicher" Existenzen (D.05, 13. April 2016; D.10) oder aber um nicht mehr als eine Unterstützung von kleineren, nicht als terroristisch zu wertenden Splittertruppen in ihrem Kampf gegen Assad, wie beispielsweise der JaS (D.04, 31. März 2017; D.02), nicht jedoch des „Islamischen Staates“ und dies mit dem Ziel der Beförderung eines friedvollen Lebens der dort 
lebenden Muslime und zur Beendigung der Gräueltaten durch in ihren Augen illegitime Staatsoberhäupter (D.08, 26. Oktober 2016; D.06).

- In der SprecherInnenposition von sog. „Kronzeugen“ (vgl. Hardinghaus, 2014:243ff.; Kneba, 2011) wird deutlich, dass solche identitären Zuschreibungen vor Gericht auch fluide sind und sich als SprecherInnenpositionen im Koordinationsystem ,,bewegen“ können. Ihre Identitätskonstruktion im Koordinatensystem von „Islamismus“ und „Terrorismus“ beruht nach eigener Einlassung darauf, dass sie sich in einem ersten Schritt zu einer Vielzahl der personellen und tatrelevanten Strukturmerkmale bekennen. Sie gestehen im Rahmen der Anklage, vormals in ihrer identitären Ausrichtung und ihren Praktiken dem oberen linken Quadranten, d.h. dem islamistischen Terrorismus, zugehörig gewesen zu sein (H.01, 22. Juni 2016; D.03). Nun befänden sie sich allerdings im Zuge einer auf radikaler Läuterung beruhenden Identitäts-Verrückung auf einem nicht-islamistischen, nicht-terroristischen Weg zurück in die hiesigen Wert-, Demokratie- und Moralvorstellungen westlicher Prägung. Trainiert und konditioniert durch die vielen Verhöre der zuständigen Ermittlungsbehörden für die Bereiche des Sagbaren, versuchen sie zur Plausibilisierung dieses Gesinnungswandels mit den oben angesprochenen Identitäts-Markern zu brechen und ihre Glaubwürdigkeit nicht nur diskursiv, sondern auch visuell-materiell sichtbar zu machen. Sie sind also nicht nur um Mithilfe bei der Verfahrensaufklärung bemüht, sondern präsentieren sich rasiert, ohne Bart, statt der T-Shirts und Hoodies, die die übrigen Angeklagten tragen, tragen sie einen Anzug, zumindest jedoch Hemd, Pullover und Blue Jeans, eine seriöse Brille unterstreicht den Gesamteindruck (D.09, 23. November 2016; D.01). Sie, die Kronzeugen, liefern die für eine Strafverfolgung bzw. Verurteilung nötigen Beweise, identifizieren andere Angeklagte, ordnen ihnen ihre Funktion in hiesigen Logistik- und Kommunikationsverbänden oder aber Positionen innerhalb der jeweiligen Kampfgruppen im fernen Syrien $\mathrm{zu}$ und bestätigen auf diese Weise $\mathrm{zu}$ einem großen Teil die in der Anklageschrift erhobenen Vorwürfe und die daraus resultierenden Verortungen als ,,islamistische TerroristInnenen“ (D.02, 11. März 2016; D.04). Sie geben sich bürgerlich, präsentieren sich als Teil einer Gesellschaft, von der sie sich vormals abgewandt hatten und in die sie nun zurück möchten. Sie präsentieren sich als Prototypen der Resozialisierbarkeit.

Auf diese Weise kommen die polarisierenden Verortungen auf den oben ausgewiesenen Identitätsachsen gewissermaßen von allen Seiten, da sowohl das Verhalten der jeweiligen Angeklagten als auch der Kronzeugen (inklusive der Interventionsbemühungen ihrer Verteidigungen) dieselben dualen Muster bedienen (müssen), die auch den Deutungen der
Taten und Asservate durch die Mitglieder des Strafsenates sowie der Generalbundesanwaltschaft zugrunde liegen. Aus diskurs- und identitätstheoretischer Perspektive ist dabei festzuhalten, dass sie sich dabei alle (trotz ihrer unterschiedlichen Verortung im Koordinatensystem der beiden Identitätsachsen) zum Zweck der jeweiligen Argumentation in die große Erzählung über den vermeintlichen Gegensatz zwischen dem Westen und den islamischen Staaten des Nahen und Mittleren Ostens einreihen (D.04, 6. Oktober 2016; D.09, 13. März 2016). Alle Seiten reifizieren durch entsprechende „Anrufungen“ die diesbezüglichen Wissensordnungen - sie praktizieren, mit Elsrud gesprochenen, ein „Othering the ,other" in court" (2014:27).

\section{Fazit}

Der vorliegende Beitrag zeigt am Beispiel von Staatsschutzverfahren gegen Mitglieder und UnterstützerInnen terroristischer Vereinigungen, dass der Gerichtssaal für die politischgeographische Forschung ein lohnenswerter Ort der Forschung sein kann, weil hier ein sehr genauer Einblick in ansonsten empirisch schwer zugängliche gesellschaftliche Spannungfelder möglich wird. Für die Herangehensweise an diesen speziellen Ort der Empirie lassen sich zunächst produktiv eine Reihe von Befunden aus der interdisziplinären Literatur nutzen, die bereits Erfahrungen mit gerichtsbezogener, ethnographischer Forschungsarbeit haben.

Der Beitrag zeigt aber gerade an seinen empirischen Beispielen auch, dass aufgrund des sehr spezifischen Diskurses autoritativer Rechtsprechung das in Gerichtsverfahren gegen mutmaßliche GefährderInnen staatlicher Sicherheit und Ordnung zu Tage tretende detaillierte Material nur dann gewinnbringend im Sinne politisch-geographisch relevanter Fragestellungen eingesetzt werden kann, wenn es konsequent durch die Perspektive entsprechender theoretischer Ansätze ausgerichtet, rekonfiguriert und re-interpretiert wird:

Es sind globalisierungstheoretische Ansätze, mit deren Hilfe sich aus der Vielfalt der gerichtlichen Einzelbefunde eine subtile raumzeitliche Choreographie des Zusammenspiels territorialer Ordnungen und Grenzregime sowie damit in komplexer Weise interagierender analoger und digitaler Netzwerke und Netzwerkbeziehungen herausarbeiten lässt. Erst eine solche Reorganisation des Blicks lässt aus den vor Gericht nach täter- und urteilsorientierten Kriterien verhandelten Asservaten und Aussagen die globalisierten Geographien des islamistischen Terrors hervortreten, die ihre Wirksamkeit gerade dadurch entfalten, dass sich, hier gezeigt an den Fällen deutscher Kämpfer und UnterstützerInnen, territorial ausgerichtete und netzwerkorientierte Logiken und Praktiken in Bereichen wie Finanzierung, Rekrutierung, Ausbildung, Ausrüstung und Einsatz in Syrien vielfältig und subtil miteinander verknüpfen.

Es sind theoretische Ansätze zur Konstruktion und Rolle raumbezogener Identitäten, die es erlauben, den Gerichtssaal 
mit Hilfe des protokollierten Materials auf seine Rolle als eine Art Identitäts-Produktions-Maschine hin zu befragen. Mit ihrer Hilfe werden Aussagen über die machtvolle Rolle (wechselseitiger) Konstruktionen geo-politischer Identitäten möglich, deren Koordinatensystem eine der wichtigen Achsen zur Konstitution des Rechtssubjekts „Terrorist“ darstellt. In diesem Sinne gibt die Analyse solcher Staatsschutzverfahren einen Einblick in die Produktionsweisen des Eigenen und des Fremden, die gerade im Rahmen einer intendierten „Wahrheitsfindung“ besonders wirkmächtig zutage treten.

Wenn der vorliegende Beitrag vor dem Hintergrund dieser einführenden und exemplarischen Befunde und Erfahrungen für eine Aufnahme von Prozessbeobachtungen in das Methoden-Portfolio humangeographischer, insbesondere politisch-geographischer Forschung plädiert, bleibt gleichwohl eine Reihe von Fragen offen, die im vorliegenden Fall in der genaueren Analyse der umfangreichen, ethnographisch erhobenen Daten adressiert werden müssen. Diese beziehen sich zum einen auf konkrete Techniken der Auswertung, wofür die bereits länger mit der Gerichtsforschung beschäftigten Nachbardisziplinen interessante Ansätze anbieten. Sie beziehen sich aber insbesondere auch auf Erweiterungen auf theoretisch-konzeptioneller Ebene, wo z.B. gerade die Berücksichtigung von Aspekten wie Materialität und/oder Emotionen/Affekten eine selektive Erweiterung des konzeptionellen Blickwinkels empfiehlt.

Datenverfügbarkeit. Vor dem Hintergrund des Schutzes von Persönlichkeitsrechten und zahlreicher, auch vertraulicher Informationen, sind die zugrunde liegen Daten im Institut für Geographie analog archiviert und können dort auf Nachfrage eingesehen werden.

Interessenkonflikt. Die Autoren erklären, dass kein Interessenkonflikt besteht.

Danksagung. Eine erste Fassung der vorliegenden Argumentation wurde auf der Tagung Neue Kulturgeographie 2016 vorgestellt. Wir bedanken uns bei allen TeilnehmerInnen für ihre konstruktiven Anmerkungen. Unser besonderer Dank gilt den anonymen GutachterInnen der Geographica Helvetica sowie Benedikt Korf als Editor, die dem Beitrag mit ihren sehr konstruktiven und weiterführenden Anmerkungen noch einmal wichtige Impulse gegeben haben.

Edited by: Benedikt Korf

Reviewed by: two anonymous referees

\section{Literatur}

Abou-Taam, M. und Sarhan, A.: Struktur und Wandel der salafistischen Szene in Deutschland, in: Salafismus und Dschihadismus in Deutschland, Herausgeber: Biene, J. und Junk, J., 25-30, Epubil, Berlin, 2016.
Atkinson, J. M.: Ethnomethodological Approaches to Socio-Legal Studies, in: Sociological approaches to law, Herausgeber: Podgórecki, A. und Whelan, C. J., 201-223, London, 1981.

Belina, B.: Predictive Policing, Monatsschrift für Kriminologie und Strafrechtsreform, 99, 85-100, 2016.

Ben Slama, B.: Die radikalisierende Wirkung von extremistischer Internetpropaganda, in: Salafismus und Dschihadismus in Deutschland, Herausgeber: Biene, J. und Junk, J., 73-76, Epubil, Berlin, 2016.

Brenner, N.: Tausend Blätter, in: Politics of scale, Herausgeber: Wissen, M., Röttger, B. und Heeg, S., 57-84, Westfäl. Dampfboot, Münster, 2008.

BKA, BfV und HKE: Analyse der Radikalisierungshintergründe und -verläufe der Personen, die aus islamistischer Motivation aus Deutschland in Richtung Syrien oder Irak ausgereist sind, online aufrufbar: https://www.verfassungsschutz.de/de/download-manager/ _analyse-der-radikalisierungshintergruende-fortschreibung-2016. pdf, letzter Zugriff: 19 November 2016.

BT-Drucksache 18/8382 vom 10.05.2016: Antwort der Bundesregierung auf die Kleine Anfrage der Abgeordneten Ulla Jelpke, Franz Tempel, Jan van Aken, weiterer Abgeordneter und der Fraktion DIE LINKE (Einschleusung von IS-Kämpfern unter die Fluchtmigration), online aufrufbar: http://dip21.bundestag. de/dip21/btd/18/083/1808382.pdf, letzter Zugriff: 12 Oktober 2016.

Burridge, A. und Gill, N.: Conveyor-Belt Justice: Precarity, Access to Justice, and Uneven Geographies of Legal Aid in UK Asylum Appeals, Antipode, 49, 23-42, 2016.

Buschauer, R.: Mobile Räume, transcript Verlag, Bielefeld, 2010.

Buzan, B. und Hansen, L.: International security: Debating security and strategy and the impact of 9-11, SAGE Publications, Los Angeles, 2007.

Castells, M.: Der Aufstieg der Netzwerkgesellschaft, VS Verlag für Sozialwissenschaften, Wiesbaden, 2001.

Cesari, J.: Securitization of Islam in Europe, Die Welt des Islams, 52, 430-449, 2012.

Clément, M.: Zwischen Banalisierung und Dramatisierung, in: Salafismus und Dschihadismus in Deutschland, Herausgeber: Biene, J. und Junk, J., 91-97, Epubil, Berlin, 2016.

Cowen, D.: The Deadly Life of Logistics, University of Minnesota Press, Minneapolis, 2014.

El-Mafaalani, A., Fathi, A., Mansour, A., Müller, J., Nordbruch, G. und Waleciak, J.: Ansätze und Erfahrungen der Präventions- und Deradikalisierungsarbeit, HSFK-Report, Frankfurt am Main, 6, 2016.

Elden, S.: Terror and Territory, Antipode, 39, 821-845, 2007.

Elsrud, T.: Othering the "other" in court, International Journal of Law, Language Discourse, 4, 27-68, 2014.

Foucault, M.: Archäologie des Wissens, 17. Auflage, Suhrkamp Verlag, Frankfurt am Main, 1981

Füller, H. und Glasze, G.: Sicherheit, Prävention und Verortung, Geographische Rundschau, 66, 4-7, 2014.

Garland, D.: Culture of Control: Crime and Social Order in Contemporary Society, University Press, Oxford, 2001.

Gemein, G. und Redmer, H.: Islamischer Fundamentalismus, Aschendorff Verlag, Münster, 2005.

Gregory, D.: The black flag: Guantánamo Bay and the space of exception, Geogr. Ann. B, 88, 405-427, 2006. 
Gregory, D.: Dis/Ordering the Orient, in: Orientalism and War, Herausgeber: Barkawi, T. and Stanski, K., 150-175, Oxford University Press, New York, 2013.

Gregory, D.: Vanishing points: Law, violence and exception in the global war prison, in: Violent Geographies fear, terror and political violence, ders., 1-46, Routledge, New York, 2017.

Hannah, M.: Torture and the Ticking Bomb: The War on Terrorism as a Geographical Imagination of Power/Knowledge, Ann. Assoc. Am. Geogr., 96, 622-640, 2006.

Hannken-Illjes, K., Scheffer, T. und Kozin, A.: Criminal Defense and Procedure, Comparative Ethnographies in the United Kingdom, Germany and the United States, Space and Culture, 12, 183-204, 2009.

Hardinghaus, A.: Strafzumessung bei Aufklärungs- und Präventionshilfe, Herbert Urtz Verlag, München, 2014.

Heinke, D. H.: German Jihadists in Syria and Iraq: An Update, ICSR, online aufrufbar: http://icsr.info/2016/02/ icsr-insight-german-jihadists-syria-iraq-update/ (letzter Zugriff: 4. April 2017), 2016.

Hoffmann, L.: Der Fall des Rechts und wie er zur Sprache kommt, in: Der Fall, Herausgeber: Oberzaucher, F., Bergmann, J. D. und Dausendschön-Gay, U., 287-344, Transcript Verlag, Bielefeld, 2014.

Hummel, K., Kamp, M. und Spielhaus, R.: Herausforderungen der empirischen Forschung zu Salafismus. Bestandsaufnahme und kritische Kommentierung der Datenlage, HSFK-Report, 1, Frankfurt am Main, 2016.

Husseini de Araújo, S.: Jenseits vom "Kampf der Kulturen“, Transcript Verlag, Bielefeld, 2011.

Klamt, M.: Verortete Normen, VS Verlag für Sozialwissenschaften, Wiesbaden, 2007.

Korf, B.: Geographie des Ernstfalls, Geogr. Z., 97, 151-167, 2009.

Kneba, N.: Die Kronzeugenregelung des §46b StGB., Duncker \& Humblot, Berlin, 2011.

Laclau, E. und Mouffe, C.: Hegemony and Socialist Strategy, Verso, London/New York, 1985.

Latour, B.: Science in Action, Harward University Press, Cambridge, 1987.

Latour, B.: La fabrique du droit, La Découverte, Paris, 2004.

Lautmann, R.: Justiz - die stille Gewalt, VS Verlag für Sozialwissenschaften, Wiesbaden, 2011.

Leitner, H.: The Politics of Scale and Networks of Spatial Connecitivity, in: Scale and Geographic Inquiry: Nature, Society, and Method, Herausgeber: Sheppard, E. and McMaster, R. B., 236255, Wiley-Blackwell, Oxford, 2004.

Lohlker, R., El Hadad, A., Holtmann, P. und Prucha, N.: Transnationale Aspekte von Salafismus und Dschihadismus, HSFK Report, 5, Frankfurt am Main, 2016.

Macé, É.: Terroranschläge in Frankreich seit 1995: ein postkoloniales Drama, sub urban zeitschrift für kritische stadtforschung, 3, 129-134, online aufrufbar: http://zeitschrift-suburban.de/sys/ index.php/suburban/article/view/181/290 (letzter Zugriff: 15 Oktober 2016), 2015.

Massey, D.: A global sense of place, Marxism Today, 38, 24-28, 1991.

Mattissek, A., Reuber, P. und Pfaffenbach, C.: Methoden der empirischen Humangeographie. Das Geographische Seminar. 2. überarbeitete und ergänzte Auflage, Westermann, Braunschweig, 2013.
Molthagen, D.: Die offene Gesellschaft im Zangengriff, in: Salafismus und Dschihadismus in Deutschland, Herausgeber: Biene, J. und Junk, J., 139-144, Epubil, Berlin, 2016.

Nacos, B. L.: Terrorism and Counterterrorism, 4. Edition, Pearson, Glenview, 2011.

Neumann, P.: Die neuen Dschihadisten. ISIS, Europa und die nächste Welle des Terrorismus, Econ, Berlin, 2015.

Pates, R., Dölemeyer, A. und Leser, J.: Schwierige Verhältnisse: Menschenhandelsopfer und Geschlecht in Gerichtsverfahren, Femina Politica, 25, 24-38, 2016.

Pollner, M.: Mundane reason: reality in everyday and sociological discourse, Cambridge University Press, New York, 1987.

Pound, R.: Law in Books and Law in Action, American Law Review, 44, 12-36, 1910.

Reuber, P.: Heimat in der Großstadt. Eine sozialgeographische Studie zu Raumbezug und Entstehung von Ortsverbindungen am Beispiel Kölns und seiner Stadtviertel, Kölner Geographische Arbeiten, 58, Köln, 1993.

Reuber, P.: Politische Geographie, UTB, Paderborn, 2012.

Reuber, P. und Strüver, A.: Der Anschlag von New York und der Krieg gegen Afghanistan in den Medien - Eine Analyse der geopolitischen Diskurse, in: Die Politik räumlicher Repräsentation, Vol. 6, Herausgeber: Dzudzek, I., Reuber, P. und Strüver, A., 179-216, Forum Politische Geographie, Berlin/Münster, 2012.

Reuber, P. und Wolkersdorfer, G.: Clash of Civilization aus der Sicht der kritischen Geopolitik, Geographische Rundschau, 54, 24-29, 2002.

Said, E.: Orientalism, Pantheon, New York, 1978.

Scheffer, T.: Ethnographie mit System am Beispiel von Englischen Strafverfahren, in: Die Methodologien des Systems, Herausgeber: John, R., Henkel, A. und Rückert-John, J., 141-158, Opladen, 2010.

Scheffer, T.: Diskurspraxis in Recht und Politik. TransSequentialität und die Analyse rechtsförmiger Verfahren, Zeitschrift für Rechtssoziologie, 35, 223-244, 2015.

Steinberg, G.: Gutachten zur terroristischen Organisation „Islamischer Staat“ (IS), Stiftung Wissenschaft und Politik, Berlin, 2015.

Spielhaus, R.: Brauchen wir eigentlich wirklich mehr Forschung zum Salafismus?, in: Salafismus und Dschihadismus in Deutschland, Herausgeber: Biene, J. und Junk, J., 105-111, Epubil, Berlin, 2016.

Sylvestre, M.-E., Damon, W., Blomley, N. und Bellot, C.: Spatial Tactics in Criminal Courts and the Politics of Legal Technicalities, Antipode, 47, 1346-1366, 2015.

Travers, M. und Manzo, J. F.: Law in Action, Routledge, New York, 1997.

Twellmann, M.: Wozu Gerichtsethnographie?, in: Wissen, wie Recht ist, ders., 21-46, Konstanz University Press, Konstanz, 2016.

Vismann, C.: Akten, Fischer Taschenbuch Verlag, Frankfurt am Main, 2011.

Whatmore, S. J. und Thome, L. B.: Nourishing networks: alternative geographies of food, in: Globalising Food, Herausgeber: Goodmann, D. und Watts, M. J., 287-304, Routledge, New York, 1997.

Zedner, L.: Risiko, Sicherheit und Terrorismus: Drei Konzepte auf der Suche nach einer akademischen Disziplin, Kriminol. J., 10, 30-46, 2012. 
Ziegleder, D., Kudlacek, D. und Fischer, T. A.: Zur Wahrnehmung und Definition von Sicherheit durch die Bevölkerung, Forschungsforum Öffentliche Sicherheit, Schriftenreihe Sicherheit, 5, Freie Universität Berlin, Berlin, 2011. 